\section{First Draft Genome Sequence Resource of a Strain of Pseudocercospora fijiensis Isolated in North America}

\author{
Luis Amarillas, ${ }^{1}$ Mitzi Estrada-Acosta, ${ }^{2}$ Rubén G. León-Chan, ${ }^{1}$ Carlos López-Orona, ${ }^{2}$ and \\ Luis Lightbourn ${ }^{1, \dagger}$ \\ ${ }^{1}$ Instituto de Investigación Lightbourn, A. C., Ciudad Jiménez, Chihuahua, México \\ ${ }^{2}$ Universidad Autónoma de Sinaloa, Culiacán, Sinaloa, México
}

\begin{abstract}
Black Sigatoka disease, caused by the fungus Pseudocercospora fijiensis, is one of the most devastating diseases of banana around the world. Fungicide applications are the primary tool used to manage black Sigatoka, but fungicide resistance in $P$. fijiensis, as in other fungal pathogens, is one of the major limitations in the efficient management and prevention of this disease. In the current study, we present the draft genome of $P$. fijiensis strain IIL-20, the first genomic sequence published from a strain of this fungus isolated in North America. Bioinformatic analysis showed putative genes involved in fungus virulence and fungicide resistance. These findings may lead us to a better understanding of the molecular pathogenesis of this fungal pathogen and also to the discovery of the mechanisms conferring fungicide resistance.
\end{abstract}

Banana is one of the most important crops in the world in terms of gross value of production, but the production of this fruit is under siege from a deadly fungus (Cheng et al. 2019). The black Sigatoka disease, or black leaf streak disease, caused by the fungal pathogen Pseudocercospora fijiensis (Mycosphaerella fijiensis M. Morelet), poses a serious threat to bananas. Infected crops with this pathogen may cause over $50 \%$ of yield losses (Landry et al. 2017). Therefore, $P$. fijiensis is a major constraint to global production of banana wherever it occurs, and the most destructive, difficult to control, and damaging pathogen of banana worldwide (Oiram et al. 2019).

The chemical management of black Sigatoka disease requires many cycles of applications of synthetic fungicides per year. Worldwide, over $30 \%$ of overall banana production costs are associated with the fungicide applications for disease control (Diaz-Trujillo et al. 2018). It is estimated that annual costs associated with the control measures are responsible for 15 to $20 \%$ of the ultimate price of bananas (Peixouto et al. 2015). Moreover, the selection pressure on natural $P$. fijiensis populations continuously reduces the fungicide sensitivity, resulting in control failures in field and unmanageable levels of disease (Arango-lsaza et al. 2016).

Besides this high cost, the excessive application of fungicides has shown significant adverse effects on human health and environmental sustainability and contributes to the global emergence of antifungal resistance. There is a critical need to promote the development of new antifungal molecules for crop protection, which requires comprehensive genetic knowledge of fungal pathogenesis (Santísima-Trinidad et al. 2018). However, little is known about the genetic determinants and pathogenic mechanisms of this fungus; therefore, research is needed to

\footnotetext{
${ }^{\dagger}$ Corresponding author: L. Lightbourn; drlightbourn@ institutolightbourn.edu.mx

First and second authors contributed equally to this work.

*The $e$-Xtra logo stands for "electronic extra" and indicates there are supplementary materials published online.
}

The author(s) declare no conflict of interest.

\section{e-Xtra*}

\section{Funding}

This work was partially supported by Consejo Nacional de Ciencia y Tecnología (CONACYT)-Mexico through project 251516. Facultad de Agronomía de la Universidad Autónoma de Sinaloa provided access to the computational infrastructure.

\section{Keywords}

disease control and pest management, genetics and resistance, mycology 
Table 1. Genome summary statistics of Pseudocercospora fijiensis strain IIL-20

\begin{tabular}{lc} 
Features & Results \\
Assembly size & $44,124,525 \mathrm{bp}$ \\
Overall GC content & $54.9 \%$ \\
Sequencing read coverage depth (fold) & $102 \times$ \\
Number of protein-coding genes & 13,583 \\
Number of tRNA-coding genes & 208 \\
Gene density (genes/Mb) & 308.1 \\
Average length of transcripts & 1,345 \\
Number of total introns & 7,234 \\
Number of total exons & 16,218 \\
\hline
\end{tabular}

better understand the disease. Genome knowledge provides the initial insights into their potential virulence mechanisms, providing the possibility to design and develop crop chemical protection that is safer and more efficient (Chen et al. 2018).

$P$. fijiensis strain IIL-20 was isolated from an infected banana plant in Chiapas, Mexico. Fungal colony purification was performed by a serial dilution technique. DNA was extracted from $\sim 1 \mathrm{~g}$ of mycelium, according to a previously described protocol (Murray and Thompson 1980). A sequencing library was constructed using the Illumina TruSeq DNA Nano library preparation method. Paired-end sequencing $(2 \times 150)$ was performed on an Illumina HiSeq 2500 platform. Raw read trimming and filtering was performed using Trimmomatic (Galaxy version 0.38.0). Reads having a Phred quality score less than 30 were removed. De novo assembly was carried out using CLC Genomics Workbench (CLCbio, Qiagen, version 20.0). The completeness of de novo assembled draft genome was evaluated using BUSCO (benchmarking universal single-copy orthologs, v3.0). Protein-coding genes were predicted using GeneMark-ES and AUGUSTUS (with the parameters of the fungal species Coccidioides immitis as member of the Leotiomycetes) programs. The protein-coding genes were annotated with Pfam and eggNOG. Carbohydrate active enzymes (CAZymes) were predicted and classified using CUPP web server (https://cupp.info/). To identify putative effectors genes, we used the following pipeline: translated amino acid sequences based on open reading frames were analyzed using SignalP (v5.0). Subsequently, TMHMM (v2.0) was used as a preliminary filter to exclude proteins with transmembrane domains, followed by EffectorP (v2.0) analysis to identify a subset of the secreted proteins that were potential effectors. Potential virulence factors of $P$. fijiensis were identified by BLASTp searches (E-value $\leq 1 \mathrm{e}-5$ ) against PHI-Database v4.8.

After adapter removal, and filtering by length and quality, we obtained 35,128,654 pairedend reads, with an average length of $140 \mathrm{bp}$. These reads were assembled into 1,918 scaffolds longer than $200 \mathrm{bp}$, with a total length of $44.1 \mathrm{Mb}$ with $54.9 \% \mathrm{GC}$ content, a scaffold N50 length of 106,393 bp, and a maximum scaffold size of 1,056,076 bp (Table 1). The assembly displayed a high overall completeness of $96.9 \%$, only $2.1 \%$ of BUSCOs were present as fragments, and $1.0 \%$ were missing.

Overall, 13,583 putative protein-encoding genes were predicted. Detailed characterization of the draft genome suggested that 580 genes predicted to encode CAZymes including 102 auxiliary activities, 23 carbohydrate esterases, 340 glycoside hydrolases, 102 glycosyl transferases, and 21 polysaccharide lyases. This result is not surprising, because the growth efficiency and aggressiveness of fungal phytopathogens are often associated with the synthesis of hydrolytic enzymes (Verma et al. 2016).

Genomic analyses identified 138 genes encoding secreted proteins, including superoxide dismutase, glycosyl hydrolases, and proteins with auxiliary activities, among them. In addition, we found 104 protein-coding genes that might be involved in damaging the physical barrier of host cell walls and in pathogenicity. Most of these genes are associated with metabolic processes including degradation enzymes for large molecules, secondary metabolism, nutrient uptake, interference with host defense strategies, necrosis inducing proteins and adaptation to different environmental stress, comprising cell redox homeostasis proteins, cellular response to DNA damage stimulus, and proteolytic enzymes.

Annotation analysis revealed multiple genes that are involved in fungicide resistance and other toxic compounds, including 71 ABC (ATP-binding cassette transporter), 46 MFS (major facilitator superfamily) transporters, three EmrB/QacA family drug resistance transporters, 35 copper resistance proteins, eight benomyl/methotrexate resistance proteins, seven oligomycin 
resistance proteins, seven amino triazoles, six acriflavine resistance proteins, and one multidrug resistance protein fnx1.

The genome sequence of $P$. fijiensis strain IIL-20 has been deposited in GenBank under BioProject number PRJNA603393, BioSample number SAMN13930950, assembly IIL_Mf_1.0, and accession number JAAEBF000000000.1.

\section{Literature Cited}

Arango-Isaza, R. E., Diaz-Trujillo, C., Dhillon, B., Aerts, A., Carlier, J., Crane, C. F., de Jong, T. V., de Vries, I., Dietrich, R., Farmer, A. D., Fortes Fereira, C., Garcia, S., Guzman, M., Hamelin, R. C., Lindquist, E. A., Mehrabi, R., Quiros, O., Schmutz, J., Shapiro, H., Reynolds, E., Scalliet, G., Souza, M., Stergiopoulos, I., Van der Lee, T. A. J., De Wit, P. J. G. M., Zapater, M.-F., Zwiers, L.-H., Grigoriev, I. V., Goodwin, S. B., and Kema, G. H. J. 2016. Combating a global threat to a clonal crop: Banana black sigatoka pathogen Pseudocercospora fijiensis (synonym Mycosphaerella fijiensis) genomes reveal clues for disease control. PLoS Genet. 12:e1006365.

Chen, S., Hao, H., Yan, X., Liu, Y., and Chu, Y. 2018. Genome-wide analysis of Mycoplasma dispar provides insights into putative virulence factors and phylogenetic relationships. G3: Genes, Genomes, Genetics 9:317-325.

Cheng, C., Li, D., Qi, Q., Sun, X., Anue, M., David, B., Zhang, Y., Hao, X., Zhang, Z., and Lai, Z. 2019. The root endophytic fungus Serendipita indica improves resistance of banana to Fusarium oxysporum f. sp. cubense tropical race 4. Eur. J. Plant Pathol. 156:87-100.

Diaz-Trujillo, C., Chong, P., Stergiopoulos, I., Cordovez, V., Guzman, M., De Wit, P., Meijer, H. J. G., Scaliet, G., Sierotzki, H., Peralta, E. L., Arango Isaza, R. E., and Kema, G. H. J. 2018. A new mechanism for reduced sensitivity to demethylationinhibitor fungicides in the fungal banana black Sigatoka pathogen Pseudocercospora fijiensis. Mol. Plant Pathol. 19:1491-1503.
Landry, C., Bonnot, F., Ravigné, V., Carlier, J., Rengifo, D., Vaillant, J., and Abadie, C. 2017. A foliar disease simulation model to assist the design of new control methods against black leaf streak disease of banana. Ecol. Modell. 359: 383-397.

Murray, M., and Thompson, W. 1980. Rapid isolation of high molecular weight plant DNA. Nucleic Acids Res. 8:4321-4326.

Oiram, F., Lopes, M., Matias, M., Braga, T., Aragão, F., Souza da Silveira, M. R., Tomaz de Oliveira, M. M. M., and de Oliveira Silva, E. 2019. Shelf-life estimation and quality of resistant bananas to black leaf streak disease during ripening. Sci. Hortic. (Amsterdam) 251:267-275.

Peixouto, Y., Bragança, C., Andrade, W., Ferreira, C., Haddad, F., Oliveira, S., Darosci Brito, F. S., Miller, R. N. G., and Amorim, E. P. 2015. Estimation of genetic structure of a Mycosphaerella musicola population using inter-simple sequence repeat markers. Genet. Mol. Res. 14:8046-8057.

Santísima-Trinidad, A., Montiel-Rozas, M., Diéz-Rojo, M., Pascual, J., and Ros, M. 2018. Impact of foliar fungicides on target and non-target soil microbial communities in cucumber crops. Ecotoxicol. Environ. Saf. 166:78-85.

Verma, S., Gazara, R., Nizam, S., Parween, S., Chattopadhyay, D., and Verma, P. 2016. Draft genome sequencing and secretome analysis of fungal phytopathogen Ascochyta rabiei provides insight into the necrotrophic effector repertoire. Sci. Rep. 6:24638. 